\title{
Prolonged occlusion of the hepatoduodenal ligament to reduce risk of bleeding and tumor spread during recipient hepatectomy for living donor liver transplantation
}

\author{
Jin-Uk Choi, Shin Hwang, Chul-Soo Ahn, Deok-Bog Moon, Tae-Yong Ha, \\ Ki-Hun Kim, Gi-Won Song, Dong-Hwan Jung, Gil-Chun Park, and Sung-Gyu Lee \\ Division of Hepatobiliary Surgery and Liver Transplantation, Department of Surgery, \\ Asan Medical Center, University of Ulsan College of Medicine, Seoul, Korea
}

\begin{abstract}
Backgrounds/Aims: Prevention of excessive bleeding during liver transplantation (LT) operations presents a major challenge. Compared to deceased donor LT, living donor LT (LDLT) is more vulnerable to bleeding because of additional dissection procedures. We herein introduce our technique for prolonged occlusion of the hepatoduodenal ligament applied to recipient hepatectomy for LDLT. Methods: Simulated assessment of splanchnic hemodynamics on prolonged occlusion of the hepatoduodenal ligament showed that patients with cirrhotic liver appeared to tolerate the procedure as like in the patients with normal liver. We accumulated experience on the prolonged Pringle maneuver with curved intestinal clamps. Results: This technique was applied to more than 60 cases of adult LDLT operations from early 2014 until the end of 2018. Initially, application of this technique was limited to patients showing heavy bleeding during perihilar mobilization. Thereafter, this technique was applied at the start of liver mobilization and stopped after complete mobilization of the retro-hepatic vena cava. Recently, this technique was also applied during dissection of the hepatoduodenal ligament. The mean total occlusion duration was $67 \pm 13$ minutes. No patient suffered from major serosal peritoneal tearing-associated bleeding or hepatic artery problems. Intentional prolonged occlusion over two hours was applied to five patients who had advanced hepatocellular carcinoma. Conclusions: We believe that prolonged occlusion of the hepatoduodenal ligament is a simple effective method to reduce intraoperative bleeding, and that it has potential benefit to reduce the risk of intraoperative tumor spread during LDLT operations. (Ann Hepatobiliary Pancreat Surg 2019;23:61-64)
\end{abstract}

Key Words: Living donor liver transplantation; Bleeding; Pringle maneuver; Portal hypertension

\section{INTRODUCTION}

In living donor liver transplantation (LDLT), every anatomical structure of the recipient liver must be meticulously dissected for reuse during graft implantation. Such surgical procedures in LDLT often lead to excessive bleeding during mobilization of the recipient native liver, especially in patients with portal hypertension or a past history of previous liver surgery. ${ }^{1,2}$

Many surgeons performing liver transplantation (LT) and hepatobiliary surgery are well acquainted with the Pringle maneuver because it effectively reduces bleeding during hepatic parenchymal transection. ${ }^{3}$ In the Pringle maneuver, repeated intermittent occlusion of the hepatoduodenal ligament for up to 15 to 30 minutes has been attempted to minimize detrimental hepatic ischemia. Unlike liver resection, hepatic ischemia of the recipient native liver does not become a matter of concern because it will be removed. We hypothesized that such a hepatic inflow occlusion could be applied during liver mobilization for LDLT when difficult dissection is anticipated. Since there is no need to shorten to the duration of inflow occlusion, it can be prolonged over a few hours.

We herein introduce our technique for prolonged occlusion of the hepatoduodenal ligament applied to recipient hepatectomy for LDLT.

Received: December 26, 2018; Revised: December 30, 2018; Accepted: January 10, 2019

Corresponding author: Shin Hwang

Department of Surgery, Asan Medical Center, University of Ulsan College of Medicine, 88 Olympic-ro 43-gil, Songpa-gu, Seoul 05505, Korea Tel: +82-2-3010-3930, Fax: +82-2-3010-6701, E-mail: shwang@amc.seoul.kr

Copyright (C) 2019 by The Korean Association of Hepato-Biliary-Pancreatic Surgery

This is an Open Access article distributed under the terms of the Creative Commons Attribution Non-Commercial License (http://creativecommons.org/ censes/by-nc/4.0) which permits unrestricted non-commercial use, distribution, and reproduction in any medium, provided the original work is properly cited. Annals of Hepato-Biliary-Pancreatic Surgery - pISSN: 2508-5778 - elSSN: 2508-5859 


\section{PATIENTS AND METHODS}

This study consisted of two parts. The first part was a simulated assessment of splanchnic hemodynamics on prolonged occlusion of the hepatoduodenal ligament in patients with cirrhotic livers. The second part was its clinical application with technical refinement.

\section{Simulative hemodynamic assessment on occlusion of the hepatoduodenal ligament in patients with cirrhotic liver}

The native liver of a recipient requiring $\mathrm{LT}$ is often cirrhotic, and development of portal hypertension and extrahepatic portal collaterals are often seen. Because of portal hypertension, it is vulnerable to induction of brisk bleeding during perihilar mobilization and dissection of the hepatoduodenal ligament. If the blood flow within the hepatoduodenal ligament is abruptly occluded, what changes happen in the splanchnic blood flow system?

In patients with normal or non-cirrhotic liver, sudden occlusion of the main portal vein results in congestion of the splanchnic flow system, by which bowel congestion can develop since there are no sizable venous collaterals. However, the portal vein pressure in such patients is not highly elevated, thus such splanchnic congestion is often well-tolerated and easily resolved after restoration of portal flow. Occlusion of the hepatic artery does not induce any noticeable systemic effect.

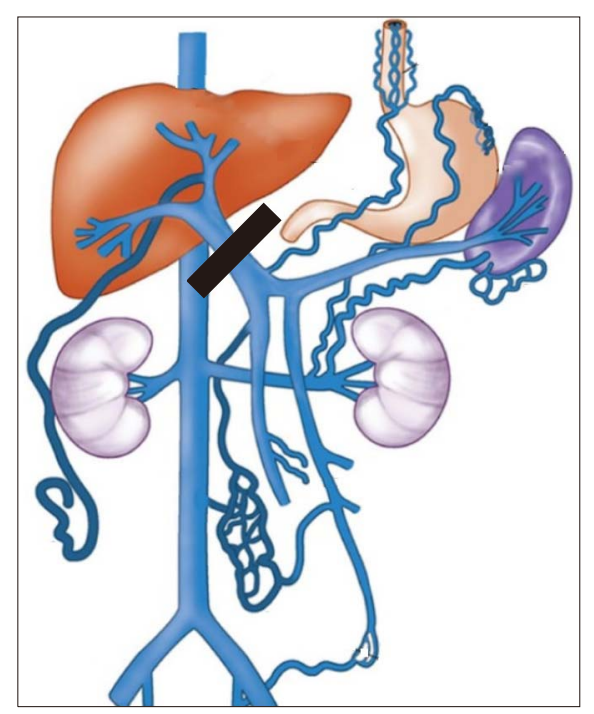

Fig. 1. Interruption of the main portal flow in a patient with liver cirrhosis and portal hypertension.
In patients with liver cirrhosis and portal hypertension, sudden occlusion of the main portal vein results in pressure increase in the splanchnic flow system, but the pressure increase is often not great because venous collaterals have already developed. Since the liver is perfused with high-pressure portal flow, any damage to the portal vein branches at the liver surface and hepatoduodenal ligament results in brisk bleeding. Complete occlusion of the hepatoduodenal ligament can cease portal vein-associated bleeding (Fig. 1). Hepatic arterial flow is also increased due to the compensatory effect on portal hypertension, thus such arterial hyperperfusion can induce excessive bleeding during perihilar mobilization and dissection of the hepatoduodenal ligament. Occlusion of the hepatic artery also prevents such risk of hepatic artery-associated bleeding (Fig. 2).

\section{Optimized technique for prolonged \\ hepatoduodenal ligament occlusion}

For the Pringle maneuver, several methods have been used, such as vascular tourniquets or vascular clamps with cover shoes, to occlude the hepatic inflow securely. Such instruments are regarded as atraumatic, but can induce tearing of the swollen or weakened hepatoduodenal ligament in the LT recipients.

We accumulated experience on the Pringle maneuver with curved intestinal clamps, thus we decided to use the

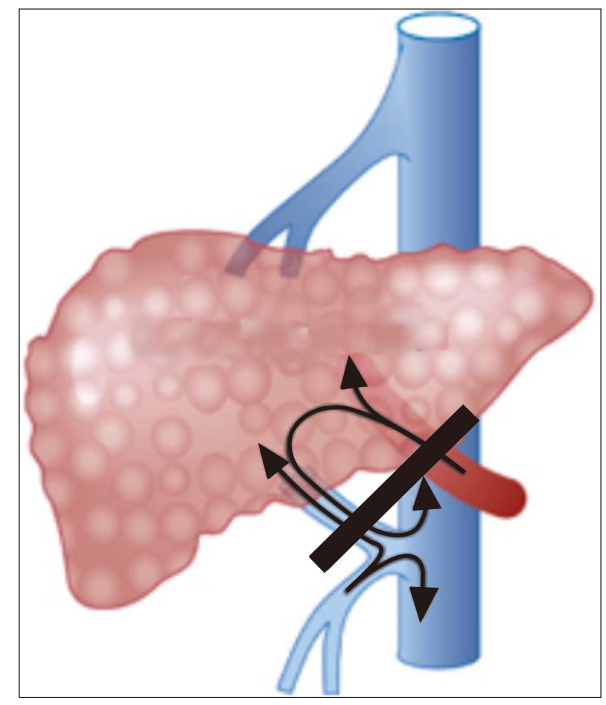

Fig. 2. Interruption of the main portal flow and hepatic arterial flow in a patient with liver cirrhosis and portal hypertension. 


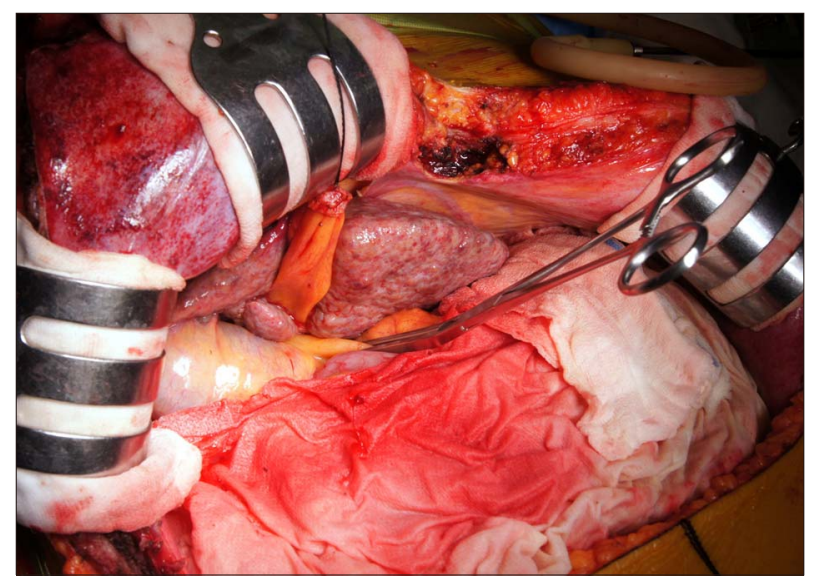

Fig. 3. Intraoperative photograph showing application of curved intestinal clamps to the hepatoduodenal ligament for right liver mobilization.

curved intestinal clamps in our study (Fig. 3). The inflow occlusion with curved intestinal clamps has the following two merits: its application and release are simple, thus it enables us to control occlusion easily; and the strength of clamping can be adjusted intuitively, thus clamping per se becomes truly atraumatic. We learned that clamping power to the first 2-3 jaw steps was enough to occlude the portal flow.

\section{RESULTS}

Between early 2014 and the end of 2018, prolonged occlusion of the hepatoduodenal ligament using curved intestinal clamps has been applied to more than 60 cases of adult LDLT.

Until the end of 2016, this technique was limited to patients showing heavy bleeding during perihilar mobilization. Application of this technique effectively reduced bleeding during mobilization of the right liver and detachment from the retro-hepatic vena cava. After a learning period, this technique was applied at the start of mobilization and stopped after complete mobilization of the retro-hepatic vena cava (Fig. 3).

Since early 2017 , this technique has also been applied during dissection of the hepatoduodenal ligament. The clamps were applied at the distal part of the hepatoduodenal ligament, and then dissection of the proximal hilum continued (Fig. 4). The clamps were temporarily released to palpate the running course of the hepatic arteries. Such a prolonged occlusion enabled us to interrupt the

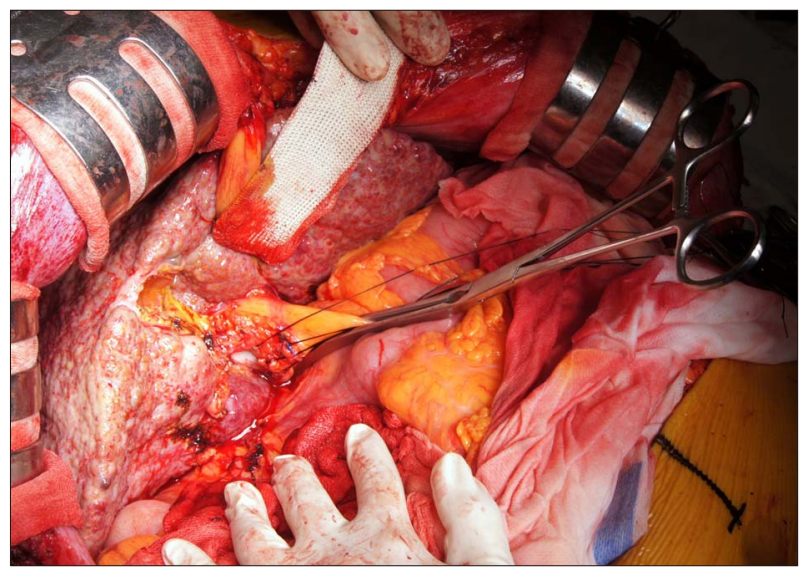

Fig. 4. Intraoperative photograph showing application of curved intestinal clamps to the hepatoduodenal ligament for proximal hilar dissection.

hepatic arterial flow effectively, by which the risk of tumor spread could be reduced theoretically. Thus, LT for advanced HCC became an eligible indication of this technique.

Analysis of the first 60 cases showed that the mean total occlusion duration was $67 \pm 13$ minutes. One-third of patients (20 cases) underwent two sessions of occlusion, the first during mobilization of the right liver and detachment from the retro-hepatic vena cava, and the second for hilar dissection. No patient suffered from major serosal peritoneal tearing-associated bleeding or hepatic artery problems. Intentional prolonged occlusion over two hours was applied to five patients who had advanced multiple hepatocellular carcinoma (HCC), and none of them showed tumor recurrence within six months after LDLT.

\section{DISCUSSION}

Prevention of excessive bleeding during LT operation presents a major challenge. We have performed more than 6000 LT operations so far, but a considerable proportion of patients still required high transfusion amounts during LT operation. In deceased donor LT, mobilization of the recipient native liver and hilar dissection can be performed promptly. In contrast, LDLT requires additional procedures for preservation of the retro-hepatic inferior vena cava and small hepatic artery branches, thus risk of bleeding inevitably increases. ${ }^{1,4,5}$

Here, we have share our surgical experiences learned from non-transplant hepatobiliary surgeries and LTs. The 
Pringle maneuver is one of the important techniques of liver surgery. James Hogarth Pringle reported his notes on the arrest of hepatic hemorrhage due to trauma in $1908 .^{6}$ Thereafter, the Pringle maneuver was settled upon as the standard procedure for liver surgery. However, as far as we know, its application was confined to liver transection alone.

We learned that the Pringle maneuver was also helpful to dissect livers heavily-adhered to the diaphragm, primarily due to previous hepatectomy. We also know that complete inflow occlusion with tight squeeze is not necessary for the Pringle maneuver. Simple application of a loose clamp may satisfy the purpose of the Pringle maneuver for reduced-bleeding hepatectomy.

In a novel move, we introduced the concept of the Pringle maneuver to the field of LT, where hepatic parenchymal transection is not necessary. Many LT surgeons intend to perform transfusion-less LT, but that goal is difficult to achieve. Various surgical techniques, including pinch-burn-cut techniques, high hilar dissection, and dissection with energy devices have been developed to reduce intraoperative bleeding during LT operations. ${ }^{7-9}$ Considering impaired coagulation profiles and concurrent portal hypertension, hemostasis in LT recipients is quite different from that of non-transplant liver surgery patients.

Our simulated hemodynamic analysis revealed that occlusion of the hepatoduodenal ligament in LT recipients can work as a stop-gap to weaken the bleeding-prone effect from portal hypertension. When a brisk bleeding occurs after sudden tearing of the venous collaterals around the liver, it is often difficult to quickly achieve local bleeding control. If the venous collateral is formed proximal to the main portal vein, hepatoduodenal ligament occlusion will work effectively. Based on our experience in more than 60 cases, we think that prolonged occlusion of the hepatoduodenal ligament is a simple and effective method to reduce intraoperative bleeding during LT operation.

Prevention of intraoperative tumor spread is another potential benefit of prolonged occlusion of the hepatoduodenal ligament. Excessive handling of the liver containing HCC can lead to hematogenous spread of the tumor into the blood stream. The surgical concept of anterior approach for large HCC was introduced clinically to reduce the risk of intraoperative tumor spread. ${ }^{10}$ Similarly, hepatoduodenal ligament occlusion can be applied during right liver mobilization with intention to minimize hematogenous spread of HCC cells in LT. This beneficial effect has not been objectively proven yet, thus we are performing a prospective study. We think that its primary indication is presence of $\mathrm{HCC} \geq 5 \mathrm{~cm}$ because this size limit is an important prognostic factor in the LDLT setting.

In conclusion, we believe that prolonged occlusion of the hepatoduodenal ligament is a simple effective method to reduce intraoperative bleeding, and has potential benefit to reduce the risk of intraoperative tumor spread during LDLT operations.

\section{REFERENCES}

1. Hwang S, Lee SG, Lee YJ, Sung KB, Park KM, Kim KH, et al. Lessons learned from 1,000 living donor liver transplantations in a single center: how to make living donations safe. Liver Transpl 2006;12:920-927.

2. Hwang S, Lee SG, Moon DB, Ahn CS, Kim KH, Lee YJ, et al. Salvage living donor liver transplantation after prior liver resection for hepatocellular carcinoma. Liver Transpl 2007;13:741746.

3. Lee KF, Wong J, Cheung SYS, Chong CCN, Hui JWY, Leung $\mathrm{VYF}$, et al. Does intermittent pringle maneuver increase postoperative complications after hepatectomy for hepatocellular carcinoma? A randomized controlled trial. World J Surg 2018;42: 3302-3311.

4. Huang CJ, Cheng KW, Chen CL, Wu SC, Shih TH, Yang SC, et al. Predictive factors for pediatric patients requiring massive blood transfusion during living donor liver transplantation. Ann Transplant 2013;18:443-447.

5. Yoon JU, Byeon GJ, Park JY, Yoon SH, Ryu JH, Ri HS. Bloodless living donor liver transplantation: risk factors, outcomes, and diagnostic predictors. Medicine (Baltimore) 2018; 97:e13581.

6. Pringle JH. V. Notes on the arrest of hepatic hemorrhage due to trauma. Ann Surg 1908;48:541-549.

7. Park YK, Kim BW, Wang HJ, Xu W. Usefulness of the Pinch-Burn-Cut (PBC) technique for recipient hepatectomy in liver transplantation. Korean J Hepatobiliary Pancreat Surg 2012;16:13-16.

8. Lee KW, Joh JW, Kim SJ, Choi SH, Heo JS, Lee HH, et al. High hilar dissection: new technique to reduce biliary complication in living donor liver transplantation. Liver Transpl 2004; 10:1158-1162.

9. Scatton O, Brustia R, Belli G, Pekolj J, Wakabayashi G, Gayet B. What kind of energy devices should be used for laparoscopic liver resection? Recommendations from a systematic review. J Hepatobiliary Pancreat Sci 2015;22:327-334.

10. Liu CL, Fan ST, Cheung ST, Lo CM, Ng IO, Wong J. Anterior approach versus conventional approach right hepatic resection for large hepatocellular carcinoma: a prospective randomized controlled study. Ann Surg 2006;244:194-203. 